\title{
Gait and balance impairments in patients with subcortical vascular cognitive impairment
}

\author{
Mahmoud Ebrahim Mostafa Elhassanien', Yasser Abo Elfotoh El-Heneedy ${ }^{1}$, Kareem Mohammed Ramadan², \\ Mona Ahmed Kotait ${ }^{3}$, Amr Elkholy ${ }^{4}$, Mohammed Youssef Elhamrawy ${ }^{5}$ and Wafik Said Bahnasy ${ }^{1 *}$ (D)
}

\begin{abstract}
Background: Subcortical vascular cognitive impairment (SVCl) is a subtype of vascular cognitive impairment associated with extensive cerebral small vessel diseases (CSVDs) imaging biomarkers. The objectives of this work were to study the existence and patterns of gait and balance impairments in patients with SVCI due to CSVDs.

Methods: The study was conducted on 28 newly diagnosed SVCI patients and 22 healthy control subjects (HCS) submitted to the advanced activity of daily living scale (AADLs), Berg balance test (BBT), Montreal Cognitive Assessment Scale (MoCA), computerized dynamic posturography (CDP), vision-based 3-D skeletal data gait analysis, and brain MRI volumetric assessment.

Results: SVCI patients showed a significant decrease in AADLs as well as total cerebral white matter volume, total cerebral cortical volume, and mean cortical thickness which were proportional to the degree of cognitive impairment as measured by the MoCA score. Regarding CDP analysis, patients with SVCl revealed prolongation of cancelation time and spectral power for mid- and high frequencies in dynamic positions. In respect to gait analysis, there were significant decreases in mean stride length and mean cadence as well as increases in mean step width and left to right step length difference in the SVCl group compared to HCS while doing a single task. These variables get highly significant during the dual-task performance with a $p$ value $<0.001$ for each one.

Conclusion: Patients with SVCI suffer from gait and balance impairments that are proportional to the severity of their cognitive decline and greatly impair their ADLs.
\end{abstract}

Keywords: Subcortical vascular cognitive impairment, Posturography, Gait analysis, Brain MRI volumetry

\section{Introduction}

Vascular cognitive impairment (VCI) is an umbrella term that describes a group of disorders in which the vascular component is either a cause or a contributor to cognitive decline. It is the second most common cause of dementia after Alzheimer's disease with a prevalence of $1-4 \%$ in those $>65$ years [1]. VCI may evolve rapidly after a clinically evident stroke (strategic single lacunar infarct or intracerebral hemorrhage) or progresses

\footnotetext{
* Correspondence: wafiq.elbahnasi@med.tanta.edu.eg

${ }^{1}$ Neuropsychiatry Department Faculty of Medicine, Tanta University, Tanta 31527, Egypt

Full list of author information is available at the end of the article
}

insidiously due to occult subclinical vascular brain injury [2]. The latter is termed subcortical vascular cognitive impairment (SVCI) or subcortical ischemic vascular dementia and is characterized by cognitive impairment associated with extensive cerebral small vessel disease (CSVD) markers, including lacunar brain infarcts (LBIs) as well as white matter hyperintensities (WMHs) [3]. Cognitive impairment due to SVCI is location dependent, but the most affected domains include complex attention and frontal executive functions with relative sparing of episodic memory and delayed recall [4].

Gait initiation starts in the frontal lobe while effortlessly integrated gait requires intact brain networks 
ensuring fast impulses transmission. The tinny CSVDs lesions gradually disrupt brain circuits with consecutive reduction of their functional and effective connectivity progressing to disconnection syndrome [5]. Many studies were directed to the correlation between cognitive impairment and imaging changes in SVCI patients with little attention to gait and balance impairments [6]. However, computerized motion analysis of SCVI patients revealed many abnormalities during the precognitive as well as the early phases of the disease which differ from physiological aging. These gait biomechanical changes could be used as objective diagnostic and prognostic biomarkers of SVCI as well as better understanding the disrupted circuits involved in gait abnormalities [7].

\section{Aim of the work}

Aim of the work was to study the existence and patterns of gait and balance impairments in patients with newly diagnosed SVCI due to CSVDs.

\section{Methods}

This work was a randomized cross-sectional study conducted on 28 newly diagnosed SVCI patients recruited from the neurology outpatient clinic in the period from the 1st of December 2017 till the end of December 2019 diagnosed according to The Vascular Behavioral and Cognitive Disorders (VASCOG) criteria [8]. The initial studied sample was 64 SVCI patients while 36 patients were uninvolved due to either the presence of exclusion criteria or refusal of enrollment in the study. Twentytwo age- and sex-matched healthy control subjects (HCS) with normal cognitive performance were also included in the study.

The study protocol was approved by the Research Ethics Committee and Quality Assurance Unit (approval code: $31869 / 11 / 17$ ), participation in the study was voluntary, and written informed consents were approved by all participants' or their guardians for any possible risks before engagement in the study.

Studied subjects were submitted to the advanced activities of daily living scale (AADLs) [9], the Montreal Cognitive Assessment Scale (MoCA) Arabic Version [10] to estimate global cognitive functions.

Exclusion criteria encompassed patients with histories of clinically manifest strokes, definite lateralizing signs, severe cognitive impairment (MoCA $<18$ points), manifest spinal or orthopedic problems, clinically evident peripheral neuropathy, preexisting chronic medical problems, MRI contraindications, and long-term use or abuse of medications affecting cognition and/or balance.

Regarding balance examination, the Berg Balance Test (BBT) [11] was used for its clinical evaluation while computerized dynamic posturography (CDP) was performed for digital balance assessment. A wavelet analysis was applied to study the frequency of the CoM displacements used the Balance Quest, Micromedical Technologies, Multitest Equilibre 6.1.37.0, provided by Framiral, Cannes, France with static/dynamic platform. Both cancelation time (CT) and spectral power (SP) were assessed for low, mid-, and high frequency in the 3 dynamic positions ( $D$ : eyes open sway-referenced platform, E: eyes closed, sway-referenced platform, F: eyes open sway referenced surround and platform) [12] (Additional file 1).

Gait analysis was performed using the vision-based three-dimensional skeletal data method [13]. The walking trajectory was 3-rounds walking with comfortable speed, each were 3 meters in length (the camera shooting range). The subject was asked to turn in the right direction between the 1st and 2nd trips and to the left side between the 2 nd and 3rd ones. After a latent period of $10 \mathrm{~min}$, the trial was repeated but at that time, the subject was asked to carry a tray with 2 glasses of water to examine dual-task performance. Walking was analyzed regarding the mean stride length, cadence (number of steps/min), mean step width, and mean right to left step length difference.

Brain MRI images were performed using 1.5-Tesla, General Electric Scanner with quadrature 8 channels head coil, GE Healthcare, Milwaukee, WI, USA. White matter hyperintensities were graded regarding the visually graded age-related white matter changes (ARWMC) scale [14]. Studied subjects also underwent cortical reconstruction/automatic volumetric segmentation for estimation of total cerebral white matter volume (TCWM $\mathrm{V})$, total cerebral cortical volume (TCCV), and mean cortical thickness (MCT) with the free surfer image analysis suite, used both intensity and continuity information from the entire 3-dimensional MR volumes in segmentation and deformation procedures to produce representations of cortical thickness $[15,16]$.

Statistical analysis was performed using SPSS Prism, version 20, 2013 created by IBM, Illinois, Chicago, USA. $P$ value $\leq 0.05$ was considered statistically significant. Categorical variable differences were analyzed using Chisquare while numerical ones were analyzed by Student $t$ test. Pearson's test was used for correlation analysis.

\section{Results}

The study included 28 SVCI patients aged $64.35 \pm 3.91$, 15 (53.6\%) were males and their MoCA score was $21.39 \pm 1.75$. The results showed a significant increase in the incidence of hypertension in the SVCI group compared to HCS with a $p$ value of 0.006 . On the other hand, other studied vascular risks including diabetes, smoking, dyslipidemia, ischemic heart diseases, and obesity revealed a non-significant increase in their 
incidence in SVCI compared to HCS groups with $p$ value $>0.05$ (Table 1 ).

The main self-reported complaints and indications of brain MRI in the SVCI group were subjective cognitive decline, dizziness, tinnitus, decreased usual ADLs, slowed gait, repeated unintentional falls, fatigue, and depressive symptoms. In respect to MRI results of the

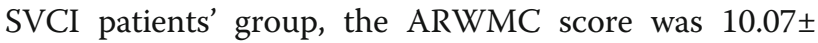
3.46 points and the mean number of occults brain lacunes was $1.07 \pm 0.72$. In respect to ADLs, there was a significant reduction in AADLs in the SVCI group compared to HCS with a $p$ value of 0.025 . Regarding the volumetric brain MRI studies, there were significant decreases in each of TCCV, TCWMV, and MCT in the SVCI group relative to the HCS ( $p$ values $<0.001$ for each) (Table 2, Fig. 1).

In respect to balance assessment, the study showed a non-significant difference between both studied groups regarding $\mathrm{BBT}$. Regarding the static $\mathrm{CDP}$, there were significant decreases of VIS and VEST ratios in the SVCI group compared to HCS with $p$ values $<0.001$ while the SOM ratio showed a non-significant difference ( $p$ value $=0.105)$. At the same time, CDP frequency (temporal) analysis identified a significant increase in each of CT and SP for mid-and high frequency in the 3-studied dynamic positions (D, E, and F) in SVCI group compared to HCS with $p$ values $<0.001$ while CT and SP for low frequencies showed non-significant differences (Table 3).

Regarding the results of gait analysis, the study showed significant decreases in mean stride length and mean cadence as well as significant increases in mean step width and left to right step length difference in the SVCI group compared to HCS while doing a single task ( $p$ values are $0.023,0.032,0.013$, and 0.002 respectively). At the same time, the differences between both groups in these studied variables get highly significant during the dual-task performance with $p$ value $<0.001$ for each one (Table 4).

Table 1 Demographic data and vascular risks among subcortical vascular cognitive impairment (group I) and healthy control subjects (group II)

\begin{tabular}{llll}
\hline & $\begin{array}{l}\text { Group I } \\
(n=28)\end{array}$ & $\begin{array}{l}\text { Group II } \\
(n=22)\end{array}$ & $p$ value \\
\hline Age & $64.35 \pm 3.91$ & $61.80 \pm 6.61$ & 0.096 \\
Male sex (male\%) & $15(53.6 \%)$ & $13(59.1 \%)$ & 0.696 \\
BMI (kg/m $\left.{ }^{2}\right)$ & $34.2 \pm 5.6$ & $32.9 \pm 6.3$ & 0.444 \\
Hypertension (\%) & $21(75 \%)$ & $8(36.4 \%)$ & $0.006^{*}$ \\
Diabetes (\%) & $14(50 \%)$ & $6(27.3 \%)$ & 0.103 \\
Smokers (\%) & $12(42.9 \%)$ & $5(22.7 \%)$ & 0.136 \\
IHD (\%) & $9(32.1 \%)$ & $5(22.7 \%)$ & 0.462 \\
\hline
\end{tabular}

IHD Ischemic heart diseases

*Significant
The results of the present study revealed negative correlations between the ARWMC score and each of AADLs and MoCA with $p$ values $<0.001$. At the same time, ARMWC showed a positive correlation with CT and SP for mid- and high frequency in the 3-studied dynamic positions (D, E, and F) as well as mean step width in dual-task performance but a significant negative correlation with TCWMV and MCT in brain volumetric studies as well as the mean cadence in dual-task gait analysis. On the other hand, the MoCA scale showed negative correlations with CT and SP for mid- and high frequency in the 3-studied dynamic positions $(\mathrm{D}, \mathrm{E}$, and F) as well as mean step width in dual-task performance (Fig. 2). The MoCA scale also showed significant positive correlations with the TCCV, TCWMV, and MCT as well as the mean cadence in dual-task gait analysis (Table 5, Fig. 3).

\section{Discussion}

Vascular cognitive impairment (VCI) is the second most common cause of acquired cognitive impairment, after Alzheimer's disease [17]. This study revealed a significantly higher incidence of hypertension in SVCI while other studied vascular risk factors (diabetes, dyslipidemia, obesity, smoking, and ischemic heart diseases) showed a non-significant increase in their incidence in SVCI patients compared to age- and sex-matched HCS. These results are passing with that of Wardlaw and colleagues [18] as well as Croall and colleagues [19] who stated that hypertension is a major risk of CSVDs and their associated VCI. On the other hand, Yu and colleagues [20] as well as Walsh and colleagues [21] found significant associations between the existence of type 2 DM as well as obesity and SVCI possibly due to the inclusion of metabolic syndrome and post-stroke cognitive impairments patients who were beyond the scope of this study. Power and colleagues [22] found a significant correlation between smoking and WMHs and VCI progression but this discrepancies in results are possibly due to different studies designs as well as smoking habits, racial, and social discrepancies.

The results of this work identified a noticeable reduction of ADLs in SVCI subjects compared to age- and sex-matched healthy control. At the same time, the study showed that some patients did not seek medical advice until the existence of a profound cognitive impairment, manifest gait impairment, and repeated unintentional fall due to the slow evolution of these disturbances and their assumption that these manifestations were due to the inevitable effect of physiological aging. These results are in harmony with the works of Cornelis and colleagues [23] as well as Madden and colleagues [24] who stated that CSVDs are neither silent nor innocent, but they are associated with a profound 
Table 2 Comparison between subcortical vascular cognitive impairment (group I) and healthy control subjects (group II) regarding MoCA, AADLs, and brain MRI findings

\begin{tabular}{|c|c|c|c|c|}
\hline & \multirow{2}{*}{$\begin{array}{l}\text { Group-I } \\
(n: 28)\end{array}$} & \multirow{2}{*}{$\begin{array}{l}\text { Group-II } \\
(n: 22)\end{array}$} & \multicolumn{2}{|l|}{$t$ test } \\
\hline & & & $t$ value & $p$ value \\
\hline MoCA & $21.39 \pm 1.75$ & $26.41 \pm 0.73$ & 12.582 & $<0.001^{*}$ \\
\hline AADLs & $25.96 \pm 4.25$ & $28.50 \pm 3.29$ & 2.307 & $0.025^{*}$ \\
\hline BBT & $45.11 \pm 2.01$ & $44.50 \pm 2.06$ & 1.049 & 0.300 \\
\hline ARWMC & $10.07 \pm 3.46$ & $2.82 \pm 1.22$ & 9.650 & $<0.001 *$ \\
\hline Occult LBIs number & $1.07 \pm 0.72$ & $0.45 \pm 0.60$ & 3.250 & $0.002^{*}$ \\
\hline Total CCV $\left(\mathrm{mm}^{3}\right)$ & $531981.8 \pm 10304.6$ & $590647.7 \pm 26713.4$ & 10.677 & $<0.001^{*}$ \\
\hline Total CWMV $\left(\mathrm{mm}^{3}\right)$ & $526508.7 \pm 12502.8$ & $587565.1 \pm 10051.4$ & 18.644 & $<0.001 *$ \\
\hline Mean cortical thickness & $2.32 \pm 0.39$ & $2.80 \pm 0.25$ & 5.067 & $<0.001^{*}$ \\
\hline
\end{tabular}

$A A D L s$ Advanced activity of daily living scale, ARWMC Age-related white matter changes scale, BBT Berg balance test, $C W M V$ cerebral white matter volume, $C C V$ cerebral cortical volume, MoCA Montreal Cognitive Assessment Scale, LBIs lacunar brain infarcts

*Significant

reduction in brain network strength ending in disconnection syndrome which will be complicated by cognitive impairment. They also stated that the decreased ADLs in patients with VCI are proportional to the degree of cognitive impairment especially executive dysfunctions. Moreover, they attributed the reduced insights of the patients concerning their gait and cognitive disturbances to their slow evolution as a result of brain resilience to network changes by undergoing several neuro-modulatory processes which reduce the costs of wiring including reorganization of the resting-state and default mode networks as well as paradoxical networks functional hyper-connectivity.

The present study identified a significant reduction in TCCV and TCWMV as well as MCT in SVCI patients which were proportional with the decline in their cognitive abilities as well as markers of CSVDs as measured by the ARWMC scale and occult LBIs number. These results are in accordance with the works of Frey and colleagues [25] as well as Loos and colleagues [26] who identified brain atrophic changes in patients with $\mathrm{VCI}$ due to CSVDs. They attributed these changes to grey and white matter volume loss due to white matter shrinkage by neuronal loss and gliosis as well as cortical thinning owing to disruption of axons traversing the WMHs lesions with resultant dieback of their neuronal cell bodies. They also found that the severities of white matter volumetric and cortical thickness reductions were proportional to the severity of CSVDs and WMHs lesions burden.

Regarding balance and equilibrium clinical assessment using the BBT, the study showed statistically nonsignificant differences between SVCI patients and HCS. This result points to the limitations of visual balance analysis in identification of subtle balance disturbances. These results are in harmony with the work of Shen and colleagues [27] who concluded that the visually rated clinical balance evaluation is an insensitive biomarker

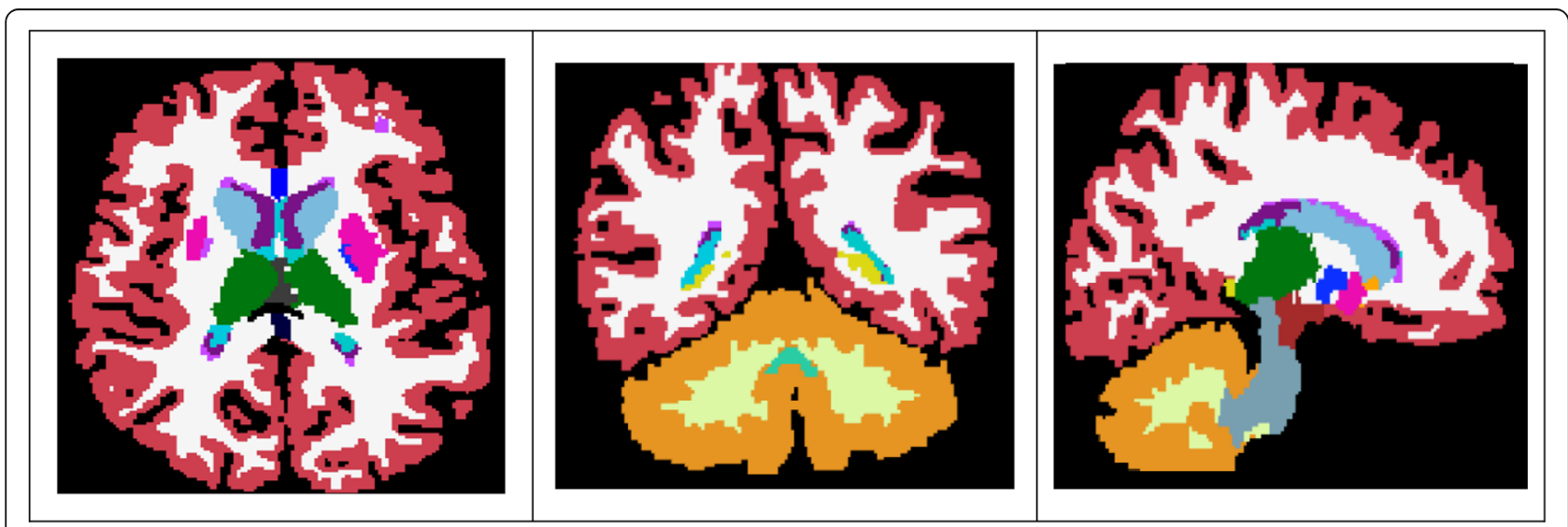

Fig. 1 Brain volumetric MRI study in a subcortical vascular cognitive impairment subject with diminished total cerebral cortical and white matter volumes as well as mean cortical thickness 
Table 3 Comparison between subcortical vascular cognitive impairment (group I) and healthy control subjects (group II) regarding computerized dynamic posturography findings

\begin{tabular}{|c|c|c|c|c|}
\hline & Group-I & Group-II & $t$ test & \\
\hline & $(n: 28)$ & (n: 22) & $t$ value & $p$ value \\
\hline CDP-SOM ratio & $92.30 \pm 11.34$ & $96.91 \pm 2.30$ & 1.623 & 0.105 \\
\hline CDP_VIS ratio & $76.27 \pm 2.89$ & $90.17 \pm 2.06$ & 13.532 & $<0.001^{*}$ \\
\hline CDP_VEST ratio & $51.65 \pm 4.18$ & $68.03 \pm 2.98$ & 20.671 & $<0.001^{*}$ \\
\hline $\mathrm{CT} / \mathrm{D}$, midfrequency & $1.42 \pm 0.59$ & $0.90 \pm 0.36$ & 3.657 & $<0.001 *$ \\
\hline$C T / D$, high frequency & $0.43 \pm 0.16$ & $0.26 \pm 0.15$ & 3.885 & $<0.001^{*}$ \\
\hline $\mathrm{CT} / \mathrm{E}$, midfrequency & $1.51 \pm 0.56$ & $0.53 \pm 0.35$ & 7.148 & $<0.001^{*}$ \\
\hline $\mathrm{CT} / \mathrm{E}$, high frequency & $0.43 \pm 0.23$ & $0.12 \pm 0.07$ & 5.910 & $<0.001 *$ \\
\hline $\mathrm{CT} / \mathrm{F}$, midfrequency & $1.15 \pm 0.66$ & $0.65 \pm 0.27$ & 3.314 & $0.002^{*}$ \\
\hline $\mathrm{CT} / \mathrm{F}$, high frequency & $0.45 \pm 0.27$ & $0.12 \pm 0.09$ & 5.357 & $<0.001 *$ \\
\hline SP/D, midfrequency & $84.79 \pm 5.10$ & $65.59 \pm 5.80$ & 12.440 & $<0.001^{*}$ \\
\hline SP/D, high frequency & $67.41 \pm 3.25$ & $47.12 \pm 5.11$ & 17.096 & $<0.001 *$ \\
\hline $\mathrm{SP} / \mathrm{E}$, midfrequency & $85.84 \pm 5.56$ & $62.94 \pm 3.23$ & 17.152 & $<0.001 *$ \\
\hline SP/E, high frequency & $74.70 \pm 7.54$ & $55.61 \pm 5.41$ & 10.009 & $<0.001 *$ \\
\hline$S P / F$, midfrequency & $84.76 \pm 4.97$ & $59.61 \pm 6.66$ & 15.299 & $<0.001^{*}$ \\
\hline SP/F, high frequency & $70.86 \pm 6.73$ & $56.58 \pm 7.39$ & 7.135 & $<0.001 *$ \\
\hline
\end{tabular}

CT cancelation time, CDP-SOM, VIS, VEST computerized dynamic posturography somatosensory, $D$ eyes open sway-referenced platform, $E$ eyes closed, swayreferenced platform, $F$ eyes open sway referenced surround and platform, SP spectral power

*Significant

for increased fall risks due to subclinical balance impairments. They explained this result by the inability of observer's eyes to identify high speed events, failure to observe the force of foot pressure and its dependence on the skills of the operator with frequent inter-rater variabilities.

Regarding digital balance assessment, the results showed impaired static balance control in SVCI patients due to impaired use of their sensory input. The main brunt was in the visual and vestibular inputs while somatosensory one was little affected. At the same time, CDP temporal analysis identified impaired dynamic balance in SVCI as evidenced by prolongation of the CT and SP for mid- and high frequencies in dynamic positions. At the same time, these dynamic balance impairments were proportional to the degree of cognitive impairment as well as the severity of CSVDs. These results are in harmony with that of Delmas and colleagues [28] as well as Tag Eldin and colleagues [29] who concluded that patients with SVCI suffer from impaired

Table 4 Comparison between subcortical vascular cognitive impairment (group I) and healthy control subjects (group II) regarding gait analysis variables in single and dual-task performance

\begin{tabular}{|c|c|c|c|c|}
\hline & \multirow{2}{*}{$\begin{array}{l}\text { Group-I } \\
(n: 28)\end{array}$} & \multirow{2}{*}{$\begin{array}{l}\text { Group-II } \\
\text { (n: 22) }\end{array}$} & \multicolumn{2}{|l|}{$t$ test } \\
\hline & & & $t$ value & $p$ value \\
\hline \multicolumn{5}{|l|}{ Single task } \\
\hline Mean stride length (cm) & $59.51 \pm 7.87$ & $64.33 \pm 6.31$ & 2.340 & $0.023^{*}$ \\
\hline Mean cadence (no. of steps/min) & $51.60 \pm 5.07$ & $54.40 \pm 3.53$ & 2.205 & $0.032^{*}$ \\
\hline Mean step width (cm) & $17.71 \pm 2.72$ & $15.95 \pm 1.86$ & 2.592 & $0.013^{*}$ \\
\hline Lt to Rt step length difference (\%) & $7.27 \pm 3.57$ & $4.67 \pm 1.19$ & 3.277 & $0.002^{*}$ \\
\hline \multicolumn{5}{|l|}{ Dual task } \\
\hline Mean stride length $(\mathrm{cm})$ & $50.04 \pm 6.73$ & $61.19 \pm 6.19$ & 6.016 & $<0.001^{*}$ \\
\hline Mean cadence (no. of steps/min) & $46.93 \pm 4.18$ & $52.60 \pm 3.39$ & 5.152 & $<0.001^{*}$ \\
\hline Mean step width (cm) & $20.25 \pm 2.80$ & $16.55 \pm 1.77$ & 5.416 & $<0.001^{*}$ \\
\hline Lt to Rt step length difference (\%) & $7.36 \pm 3.36$ & $4.87 \pm 1.14$ & 3.324 & $<0.001^{*}$ \\
\hline
\end{tabular}

$C T$ cancelation time, $D$ eyes open sway-referenced platform, $E$ eyes closed, sway-referenced platform, $F$ eyes open sway referenced surround and platform, $S P$ spectral power

*Significant 


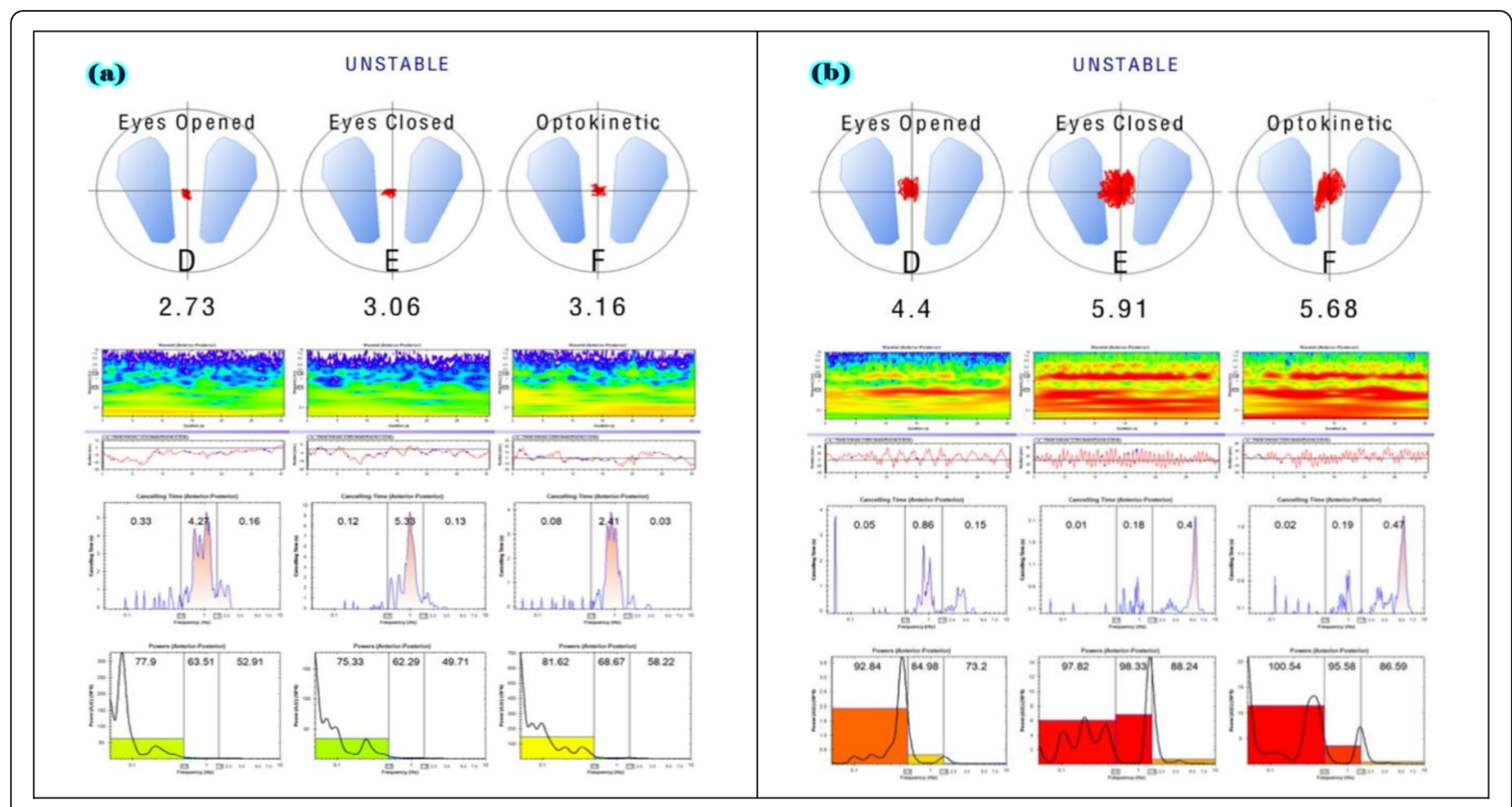

Fig. 2 Computerized dynamic posturography analysis (a) healthy control subject, (b) a patient with subcortical vascular cognitive impairment showing prolonged cancelation times and increased spectral powers in the 3 studied positions (d: eyes open sway-referenced platform, e: eyes closed, sway-referenced platform, f: eyes open sway referenced surround and platform)

postural control evidenced by decreased visual and vestibular CDP ratios as well as greater variability of center of pressure oscillation during walking. This increased fall risk is proportional to cognitive decline secondary to white matter microstructural abnormalities and reduced brain network strength.

In respect to the 3-D vision-based gait analysis, the results showed mild impairment of the assessed gait variables (mean stride length, cadence, mean step width, and mean right to left step length difference) in SVCI patients and these disturbances markedly increased while doing dual tasks. At the same time, the degree of gait disturbances especially in dual tasks was proportional with the severity of WMHs in MRI brain as well as with the severity of cognitive impairment as measured by the MoCA scale. These results are in accordance with the studies of Rosario and colleagues [30] as well as Verghese and colleagues [31] who identified the poor performance of gait parameters in patients with VCI during the early stages of the disease. They also got to as far as the introduction of the terminology motoric cognitive risk syndrome which describes gait abnormalities preceding the cognitive impairment. At the same time, Montero-Odasso and colleagues [32] studied the relation of dual-task gait and cognitive impairment which involves the performance of two concomitant tasks to assess the interference of one task on the other which may elaborate subtle abnormalities passed undetectable while performing a single task. They concluded that assessment of dual-task gait is an early biomarker for cognitive decline progression and future fall risk in patients with VCI due to CSVDs due to reduction in the attentional resources and brain network strength among such individuals.

\section{Conclusion}

Newly diagnosed SVCI patients suffer from gait and balance impairments proportional to the severity of their cognitive impairment, WMHs severities, and brain volume loss in MRI volumetric analysis. These gait and balance disturbances are associated with profound reduction ADLs as well as increased risk of unintentional falls.

\section{Recommendations}

Further studies are needed to identify the value of digital gait and balance analysis as biomarkers for cognitive impairment in patients with SVCI and CSVDs. At the same time, management trials including gait and balance rehabilitations are needed to improve these biomechanical changes and reduce risks of unintentional falls in such sector of patients.

\section{Strength}

The strength of this work is studying gait and balance performance in SVCI patients which is often underresearched and most attention is paid toward cognitive 
Table 5 Correlation analysis between each of Montreal cognitive assessment and age-related white matter changes scales with each of balance and gait variables as well as brain MRI volumetry in subcortical vascular cognitive impairment patients

\begin{tabular}{|c|c|c|c|c|}
\hline & \multicolumn{2}{|l|}{ MoCA } & \multicolumn{2}{|l|}{ ARWMC } \\
\hline & $r$ & $p$ value & $\bar{r}$ & $p$ value \\
\hline ARWMC & -0.659 & $0.001 *$ & & \\
\hline Advanced activities of daily living scale & 0.640 & $0.001^{*}$ & -0.735 & $0.001^{*}$ \\
\hline Total cortical volume & 0.460 & $0.014^{*}$ & -0.296 & 0.126 \\
\hline Total white matter volume & 0.613 & $0.001^{*}$ & -0.703 & $0.001^{*}$ \\
\hline mean cortical thickness & 0.789 & $0.001^{*}$ & -0.742 & $0.001^{*}$ \\
\hline$C T, D$, midfrequency & -0.578 & $0.001^{*}$ & 0.769 & $0.001^{*}$ \\
\hline$C T, D$, high frequency & -0.581 & $0.001^{*}$ & 0.604 & $0.001 *$ \\
\hline $\mathrm{CT}$, E, midfrequency & -0.637 & $0.001^{*}$ & 0.566 & $0.002^{*}$ \\
\hline $\mathrm{CT}, \mathrm{E}$, high frequency & -0.658 & $0.001^{*}$ & 0.769 & $0.001 *$ \\
\hline $\mathrm{CT}, \mathrm{F}$, midfrequency & -0.681 & $0.001^{*}$ & 0.792 & $0.001^{*}$ \\
\hline $\mathrm{CT}, \mathrm{F}$, high frequency & -0.769 & $0.001^{*}$ & 0.853 & $0.001^{*}$ \\
\hline SP, D, midfrequency & -0.639 & $0.001^{*}$ & 0.691 & $0.001^{*}$ \\
\hline SP, D, high frequency & -0.680 & $0.001 *$ & 0.624 & $0.001 *$ \\
\hline$S P, E$, midfrequency & -0.797 & $0.001 *$ & 0.767 & $0.001 *$ \\
\hline SP, E, high frequency & -0.826 & $0.001^{*}$ & 0.811 & $0.001^{*}$ \\
\hline$S P, F$, midfrequency & -0.786 & $0.001^{*}$ & 0.651 & $0.001^{*}$ \\
\hline SP, F, high frequency & -0.791 & $0.001 *$ & 0.753 & $0.001^{*}$ \\
\hline Mean stride length (dual task) & -0.219 & 0.086 & 0.201 & 0.304 \\
\hline Mean cadence (dual task) & 0.663 & $0.001^{*}$ & -0.588 & $0.001^{*}$ \\
\hline Mean step width (dual task) & -0.702 & $0.001^{*}$ & 0.614 & $0.001^{*}$ \\
\hline
\end{tabular}

ARWMC age-related white matter changes, $C T$ cancelation time, $D$ eyes open sway-referenced platform, $E$ eyes closed, sway-referenced platform, $F$ eyes open sway-referenced surround and platform, MoCA Montreal Cognitive Assessment scale, SP spectral power

*Significant

impairments peculiarities and progression rather than physical disabilities which may be a major cause of restricted ADLs, reduced QoLs, and unintentional falls with subsequent increased morbidity and mortality.

\section{Limitations}

Video analysis of turns regarding speed, synchrony, and the number of steps needed for a complete turn was not assessed due to the need for additional cameras and software upgrading. At the same time, the

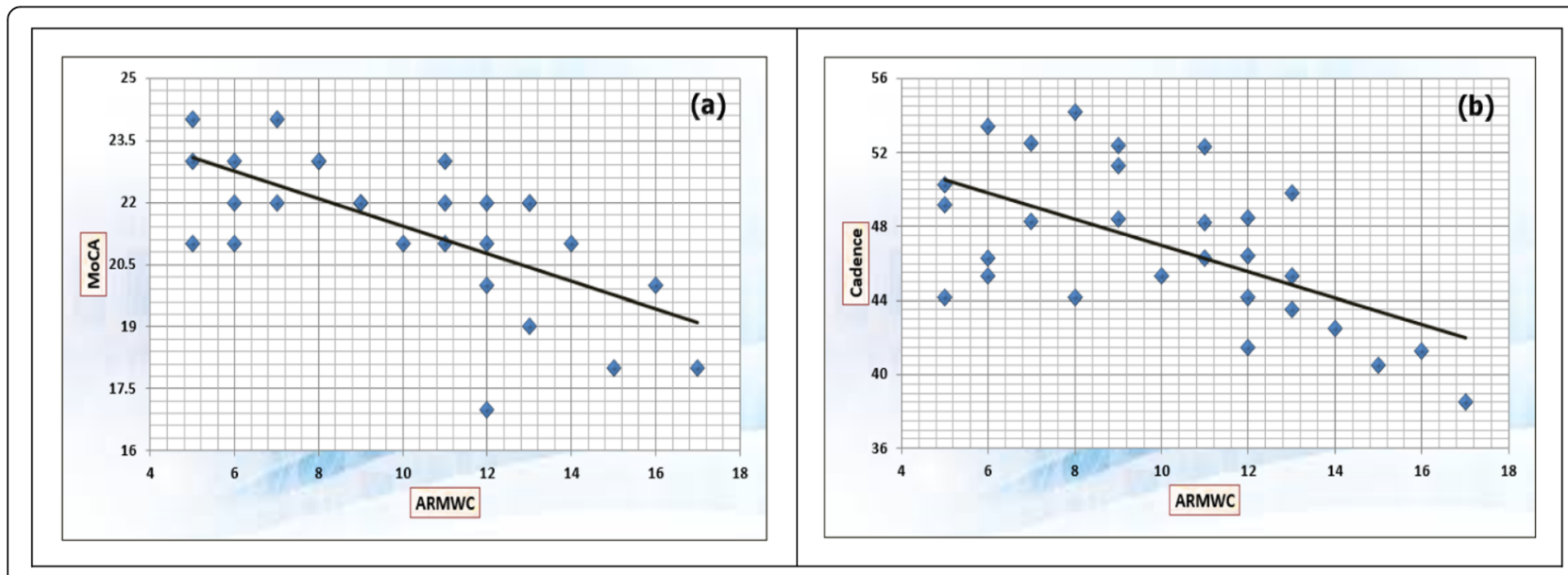

Fig. 3 A negative correlation between the ARMWC scale and each of (a) MoCA scale and (b) mean cadence in dual-task gait analysis 
small sample size for both patients and control is another item should be added to the limitations of this study.

\begin{abstract}
Abbreviations
AADLs: Advanced activities of daily living scale; ADLs: Activity of daily livings; ARWMC: Age-related white matter changes; CDP: Computerized dynamic posturography; CoM: Center of mass; CST: Corticospinal tract; CSVD: Cerebral small vessel diseases; CT: Cancelation time; LBls: Lacunar brain infarcts; MCT: Mean cortical thickness; MoCA: Montreal Cognitive Assessment Scale; SP: Spectral power; SOM: Somatosensory; SVCI: Subcortical vascular cognitive impairment; TCCV: Total cerebral cortical volume; TCWMV: Total cerebral white matter volume; $\mathrm{VCl}$ : Vascular cognitive impairment; VEST: Vestibular; VIS: Visual; WMHs: White matter hyperintensities
\end{abstract}

\section{Supplementary Information}

The online version contains supplementary material available at https://doi. org/10.1186/s41983-021-00293-5.

\begin{abstract}
Additional file 1: Computerized dynamic posturography (CDP). Figure $\left({ }^{*}\right)$. The different conditions performed in the sensory organization test; (A) eyes open, surround and platform stable, (B) eyes closed, surround and platform stable, (C) eyes open, sway- referenced surround, (D) eyes open sway-referenced platform, (E) eyes closed, sway-referenced platform and (F) eyes open sway referenced surround and platform (Quoted from Massukawa Oda and Ganança, 2015).
\end{abstract}

\section{Acknowledgements}

We would like to thank the medical and para-medical staff, Neuropsychiatry, Audiovestibular Medicine and Diagnostic Radiology Departments, Tanta University Hospitals, for their great help in patients' selection, posturography, and MRI processing.

\section{Authors' contributions}

MEME participated in the study's design, patients' selection, statistical analysis, data analysis, references collection, and manuscript writing, YAE participated in the study's idea, design, patients' selection, neurological examination, posturography assessment, statistical analysis, data analysis, references collection, manuscript writing, revision, and final approval, KMR participated in study's design, patients' assessment, MRI volumetry performance, manuscript revision, and final approval, MAK participated in study's idea and design, patients' assessment and inclusion, and posturography interpretation, data analysis, statistical analysis, manuscript writing, revision, and final approval, AE participated in study's idea and design, patients' assessment and inclusion, and gait analysis, statistical analysis, manuscript writing, revision, and final approval, MYE participated in study's idea and design, patients' assessment and inclusion, and gait analysis, manuscript writing and revision, WSB participated in the study's idea, design, patients' selection, neurological examination, posturography assessment, statistical analysis, data analysis, references collection, manuscript writing, revision, and final approval.

\section{Funding}

No funding had been received.

\section{Availability of data and materials}

The datasets used and/or analyzed during the current study are available from the corresponding author on reasonable request.

\section{Declarations}

\section{Ethics approval and consent to participate}

The manuscript was approved from The Research Ethics Committee and Quality Assurance Unit, Faculty of Medicine, Tanta University.

The URL: http://tqac.tanta.edu.eg/new-tqac/QualityAssuranceUnit@hotmail. com

Approval Code: 31869/11/17

Name of the Pl: Wafik Said Bahnasy

Name of the department: Neuropsychiatry
Type of the research: promotion research

Date of approval: November 2017

The study's protocol had permitted by The Research Ethics Committee and Quality Assurance Unit, Faculty of Medicine, Tanta University. Participations were voluntary; informed written consents were approved by all participants and any possible risks were clarified.

\section{Consent for publication}

Not applicable.

\section{Competing interests}

All authors disclose that they have no competing interests related to the study.

\section{Author details}

${ }^{1}$ Neuropsychiatry Department Faculty of Medicine, Tanta University, Tanta 31527, Egypt. 'Diagnostic Radiology Department, Faculty of Medicine, Tanta University, Tanta, Egypt. ${ }^{3}$ Audiovestibular Medicine, Faculty of Medicine, Tanta University, Tanta, Egypt. ${ }^{4}$ Computers and Automatic Control Department, Faculty of Engineering, Tanta University, Tanta, Egypt. ${ }^{5}$ Kafr El Zayat General Hospital, Ministry of Health, Al Gharbiyah, Egypt.

Received: 17 December 2020 Accepted: 26 February 2021

Published online: 10 May 2021

\section{References}

1. Rodríguez García PL, Rodríguez GD. Diagnosis of vascular cognitive impairment and its main categories. Neurología. 2015;30(4):223-39.

2. Moscufo N, Wakefield DB, Meier DS, Cavallari M, Guttmann CRG, White WB, et al. Longitudinal microstructural changes of cerebral white matter and their association with mobility performance in older persons. PLoS One. 2018;13(3):e0194051.

3. El-Senousy MY, Khalil MK, Bahnasy WS, El-Heneedy YA, Hassanien OA, ElShafey R. Leukoaraiosis as a predictor of short-term outcome of acute ischemic stroke. Egypt J Neurol Psychiat Neurosurg. 2013;50(3):239-45.

4. Zhu Z, Liao H. Impact of cognitive impairment and systemic vascular comorbidities on risk of all-cause and cardiovascular mortality: National Health and nutrition examination survey 1999 to 2002. Int J Cardiol. 2020; 300:255-61.

5. Rensma SP, van Sloten T, Launer L, Stehouwer CDA. Cerebral small vessel disease and risk of incident stroke, dementia and depression, and all-cause mortality: A systematic review and meta-analysis. Neurosci Biobehav Rev. 2018;90:164-73.

6. Langen CD, Cremers LGM, de Groot M, White T, Ikram MA, Niessen WJ, et al. Disconnection due to white matter hyperintensities is associated with lower cognitive scores. Neurolmage. 2018;183:745-56.

7. Snyder AZ, Bauer AQ. Mapping structure-function relationships in the brain. Biol Psychiatry Cogn Neurosci Neuroimaging. 2019;4(6):510-21.

8. Sachdev P, Kalaria R, O'Brien J, Skoog I, Alladi S, Black SE, et al. Diagnostic criteria for vascular cognitive disorders: a VASCOG statement. Alzheimer Dis Assoc Disord. 2014;28(3):206-18.

9. Dias EN, da Silva JV, Pais-Ribeiro JL, Martins T. Validation of the advanced activities of daily living scale. Geriatr Nurs. 2019;40:7-12.

10. Abdel Rahman TT, El Gaafary MM. Montreal cognitive assessment Arabic version: reliability and validity prevalence of mild cognitive impairment among elderly attending geriatric clubs in Cairo. Geriatr Gerontol Int. 2009;9: 54-61.

11. Berg K, Wood-Dauphinee S, Williams JI, Maki B. Measuring balance in the elderly: validation of an instrument. Can J Public Health. 1992;2:S7-11.

12. Massukawa Oda DT, Ganança CF. Computerized dynamic posturography in the assessment of body balance in individuals with vestibular dysfunction. Audiol Commun Res. 2015;20(2):89-95.

13. Elkholy A, Hussein ME, Goma W, Damen D, Saba E. Efficient and robust skeleton-based quality assessment, and abnormality detection in human action performance. IEEE J Biomed Health Inform. 2020;24(1):280-91.

14. Wahlund LO, Barkhof F, Fazekas F, Bronge L, Augustin M, Sjögren M, et al. A new rating scale for age-related white matter changes applicable to MRI and CT. Stroke. 2001;32:1318-22.

15. Reuter M, Schmansky NJ, Rosas HD, Fischl B. Within-subject template estimation for unbiased longitudinal image analysis. Neuroimage. 2012; 61(4):1402-18. 
16. Bahnasy WS, El-Heneedy YAE, Ragab OAA, Badr MY, Seleem MAH, Amer RA, et al. Polysomnography, brain volumetry, and mismatch negativity as early biomarkers of amnestic mild cognitive impairment progression. Egypt J Neurol Psychiatry Neurosurg. 2018:54:20.

17. Abd Ghafar MZA, Miptah HN, O'Caoimh R. Cognitive screening instruments to identify vascular cognitive impairment: a systematic review. Int I Geriatr Psychiatry. 2019;34:1114-27.

18. Wardlaw JM, Smith C, Dichgans M. Small vessel disease: mechanisms and clinical implications. Lancet Neurol. 2019;18(7):684-96.

19. Croall ID, Tozer DJ, Moynihan B, Khan U, O'Brien JT, Morris RG, et al. Effect of standard vs. intensive blood pressure control on cerebral blood flow in small vessel disease. The PRESERVE randomized clinical trial. JAMA Neurol. 2018;75(6):720-7.

20. Yu X, Wang G, Zhan J, Zhang Z, Feng T, XU J. Risk factors of pure leukoaraiosis and the association with preclinical carotid atherosclerosis. Atherosclerosis. 2018;275:328-32.

21. Walsh El, Shaw M, Sachdev P, Anstey KJ, Cherbuin N. The impact of type 2 diabetes and body mass index on cerebral structure is modulated by brain reserve. Eur J Neurol. 2019;26(1):121-7.

22. Power MC, Deal JA, Sharrett AR, Jack CR, Knopman D, Mosley TH, et al. Smoking and white matter hyperintensity progression. The ARIC-MRI study. Neurology. 2015;84(8):841-8.

23. Cornelis E, Gorus E, Van Schelvergem N, De Vriendt P. The relationship between basic, instrumental, and advanced activities of daily living and executive functioning in geriatric patients with neurocognitive disorders. Int J Geriatr Psychiatry. 2019;34(6):889-99.

24. Madden DJ, Parks EL, Tallman CW, Boylan MA, Hoagey DA, Cocjin SB, et al. Sources of disconnection in neurocognitive aging: cerebral white-matter integrity, resting-state functional connectivity, and white-matter hyperintensity volume. Neurobiol Aging. 2017;54:199-213.

25. Frey BM, Petersen M, Mayer C, Schulz M, Cheng B, Thomalla G. Characterization of white matter hyperintensities in large-scale MRI-studies. Front. Neurol. 2019:10;238.

26. Loos CMJ, Makin SDJ, Staals J, Dennis MS, van Oostenbrugge RJ, Wardlaw JM. Long-term morphological changes of symptomatic lacunar infarcts and surrounding white matter on structural magnetic resonance imaging. Stroke. 2018:49:1183-8.

27. Shen DC, Wu SL, Shi YZ, Wang S, Zhang YM, Wang CX. The correlation between white matter hyperintensity and balance disorder and fall risk: an observational, prospective cohort study. Chronic Dis Transl Med. 2016;2(3): 173-80.

28. Delmas S, Watanabe T, Yacoubi B, Christou EA. Age-associated increase in postural variability relates to greater low-frequency center of pressure oscillations. Gait Posture. 2020:85:103-9.

29. Tag Eldin AEA, Bahnasy WS, Dabees NL, Fayed HA. Cognitive and balance impairments in people with incidental white matter hyperintensities. Egypt J Neurol Psychiatr Neurosurg. 2020;56:97.

30. Rosario BL, Rosso AL, Aizenstein HJ, Harris T, Newman AB, Satterfield S, et al. Cerebral white matter and slow gait: contribution of hyperintensities and normal-appearing parenchyma. Gerontol A Biol Sci Med Sci. 2016;71(7):96873.

31. Verghese J, Annweiler C, Ayers E, Barzilai N, Beauchet O, Bennett DA, et al. Motoric cognitive risk syndrome: multicountry prevalence and dementia risk. Neurology. 2014;83(8):718-26.

32. Montero-Odasso MM, Sarquis-Adamson Y, Speechley M, Borrie MJ, Hachinski VC, Wells J, et al. Association of dual-task gait with incident dementia in mild cognitive impairment. JAMA Neurol. 2017;74(7):857-65.

\section{Publisher's Note}

Springer Nature remains neutral with regard to jurisdictional claims in published maps and institutional affiliations.

\section{Submit your manuscript to a SpringerOpen ${ }^{\circ}$ journal and benefit from:}

- Convenient online submission

- Rigorous peer review

- Open access: articles freely available online

- High visibility within the field

- Retaining the copyright to your article

Submit your next manuscript at $\boldsymbol{\nabla}$ springeropen.com 